\title{
A Handbook of Primary PCl: No-Reflow Management
}

\author{
Julien Adjedj, Olivier Muller, and Eric Eeckhout
}

\subsection{Introduction}

Although substantial progress has been made in recent decades in reducing mortality and performing optimal revascularization in patients with acute coronary syndrome (ACS) and stable coronary artery disease (CAD), one of the remaining challenges is to better prevent and treat extended myocardial damage despite "apparent" angiographic optimal percutaneous coronary intervention (PCI). The presence of no-reflow is related to higher risk of major adverse cardiac events (MACE) due to the poor healing of the infarct, adverse left ventricular remodelling, congestive heart failure occurrence and death. Despite optimal epicardial coronary artery reperfusion performed by PCI, distal microembolization into the coronary microcirculation limits myocardial salvage especially during ACS. No-reflow represents the ultimate stage of extended myocardial damage after PCI with absence of contrast medium progression in the coronary artery. This complication occurs mainly during ACS or during PCI of rotational atherectomy and venous graft in stable patients. The objective of this chapter is to describe how to manage a noreflow phenomenon from the pathophysiology to the management in order to help physician to prevent this complication and if no-reflow occurs adapt therapeutics to limit myocardial damage and reduce poor outcomes.

\subsection{Definition}

The no-reflow phenomenon is defined as inadequate myocardial perfusion through a given segment of the coronary circulation without angiographic evidence of mechanical obstruction [1].

J. Adjedj · O. Muller · E. Eeckhout $(\triangle)$

CHUV, Cardiology Department, Lausanne University Hospital, Lausanne, Switzerland

e-mail: eric.eeckhout@chuv.ch 


\subsection{Pathophysiology}

Understanding the pathophysiology of the no-reflow phenomenon is the key to manage this phenomenon and prevent poor outcome. After prolonged coronary occlusion and restoration of coronary blood flow, structural damage to the microvasculature reduces the amount of blood flow to the cardiac myocytes. This may lead to inadequate healing of the cardiac scar.

\subsection{Aetiology}

No-reflow phenomenon is commonly the consequence of distal embolization and reperfusion injury such as:

- Thrombus containing lesions (ACS)

- Oxygen free radical or cellular-mediated endothelial injury (ACS)

- Loss of microvascular compartment due to completed myocardial infarction

- Cellular and interstitial oedema

- Atherosclerotic debris (venous bypass graft or rotational atherectomy)

- Microvascular constriction of vasospasm (drugs, choc)

No-reflow generally occurs immediately after PCI between 1 and 3\% of PCI and can arise in different clinical settings [2-8]:

- Late presentation ACS

- Large thrombus burden

- Venous bypass graft PCI

- Rotational atherectomy

- Cardiogenic shock

\subsection{Diagnosis}

\subsubsection{Clinical Presentation}

Generally during ACS, revascularization is associated with relief of symptoms such as chest pain and regression of the ST-segment elevation in the electrocardiogram (ECG) in absence of no-reflow. In the presence of no-reflow, in the catheterization laboratory, the clinical presentation of no-reflow is often sudden and tragic. Control of coronary angiography confirms contrast medium staining in the coronary artery; the patient might complain of chest pain and symptoms persistence with a residual or increase elevation of the ST segment generally followed by haemodynamic instability. 


\subsubsection{Coronary Angiography}

Contrast medium progression speed into the coronary artery is preserved in the absence of sub occlusive coronary stenosis $(<90 \%)$ and in the absence of microcirculation damage. Therefore, after successful revascularization of an epicardial coronary stenosis, contrast medium progression impairment could reflect microcirculation damage. From the Thrombolysis in Myocardial Infarction (TIMI) study group, two indices were described: TIMI flow grade $0-3$ is a semi-quantitative variable that ranges from no contrast medium progression (0) to normal progression (3); TIMI frame count is a quantitative index calculating the number of frames between two landmarks proximal and distal to the interrogated coronary artery [9]. In patients with ACS and preserved TIMI grade flow after revascularization, microcirculation can also be evaluated with myocardial blush, which corresponds to a densitometric method, assessing maximum intensity of contrast medium in the microcirculation. In practice, coronary microvascular obstruction is defined as TIMI grade flow $\leq 3$ with myocardial blush stagnation (grade 0 or 1) [10] and no-reflow as the absence of contrast progression in the coronary vessel of interest in his most evident form. However, this may be subtle with preserved TIMI flow and absence of myocardial blush.

\subsection{Management of No-Reflow}

\subsubsection{No-Reflow Prevention: Before the Procedure}

No-reflow phenomenon is rare among overall PCI (1-3\%) although some situations are associated with higher rate of no-reflow; therefore, management of some factors could help to prevent its occurrence according to the aetiology. Traditional cardiovascular risk factors are associated with poor outcome and no-reflow increase rate. In patients with diabetes, optimal blood sugar control before the procedure could reduce the occurrence of no-reflow $[11,12]$. An animal study suggests that hypertension might be associated with increased risk of no-reflow [13]. Meta-analysis showed that pre-procedural use of statins was associated with significant reduction rate of noreflow by $4.2 \%$ in all PCI patients (risk ratio (RR) $0.56,95 \%$ confidence interval (CI) $0.35-0.90, P=0.016$ ) and attenuated by $5.0 \%$ in non-STEMI patients (RR $0.41,95 \%$ CI $0.18-0.94, P=0.035)$. This benefit was mainly observed in the early or acute intensive statin therapy populations (RR $0.43,95 \%$ CI $0.26-0.71, P=0.001$ ) [14]. Active double antiplatelet therapy will prevent PCI complications such as acute stent thrombosis, periprocedural myocardial infarction (MI) and no-reflow (Fig. 17.1).

\subsubsection{No-Reflow Prevention: During the Procedure}

General good practice of PCI could limit the no-reflow occurrence. Anticoagulation should be performed at the early phase of the procedure with unfractionated heparin 
(70-100 UI/Kg) and monitored with the activated clotting time (ACT) (200-250). Intracoronary nitrates should be administered (100-200 mcg) as well at the early phase, i.e. second angiographic view of diagnostic procedure. Optimal catheter selection is key to avoid damping intracoronary pressures, which can reduce coronary flow due to catheter induced obstruction and thereby lead to thrombus formation. Regular and systematic flushing of catheters can avoid thrombus and air emboli. Because new microthrombus composed of platelet and fibrin is an important contributor to the pathogenesis of the no-reflow phenomenon, glycoprotein IIb/ IIIa platelet receptor inhibitor (anti-GPIIb/IIIa) may be beneficial in prevention during PCI. Studies suggest that anti-GPIIb/IIIa is beneficial in reducing rates of death, reinfarction and urgent revascularization when used in conjunction with PCI particularly as a rescue strategy [15] (Fig. 17.1).

\subsubsection{Rotational Atherectomy}

Balloon angioplasty exerts beneficial effects by enlarging the weakest part of coronary artery wall thereby producing intimal splits and medial dissections in calcified lesions. In contrast, rotational atherectomy aims to weaken calcified lesions, erode calcium spicule protrusion and thereby obtains a relatively smooth luminal surface. Rotational atherectomy use a burr rotation with high speed which generate friction (microembolization) and heat (platelet activation) between the burr and calcify plaque.

In experimental modelling, heat varies with technique from $2.6{ }^{\circ} \mathrm{C}$ using intermittent ablation and permitting minimal decelerations (5000 rpm) to $13.9^{\circ} \mathrm{C}$ using continuous ablation allowing excessive decelerations (16,000 rpm) [16]. Along with microembolization of debris associated with thrombi, thermal injury may contribute to microvascular obstruction and no-reflow. To prevent these phenomena, medical therapy includes effective dual antiplatelet therapy, vasodilators and proper use of rotablational atherectomy. The benefit of antiplatelet therapy was established with the use of anti-GPIIb/IIIa reducing procedural morbidity and CK-MB elevation [17]. Preventive vasodilators are used for the purpose of reducing slow-flow and no-reflow, combining nitrates with calcium inhibitors and sometimes adenosine in the flush solution associated with heparin. Nicardipine may be effective when administered in a flush solution with other drugs during rotational atherectomy [18]. Recommended manipulation of the rotational atherectomy to reduce the risk of

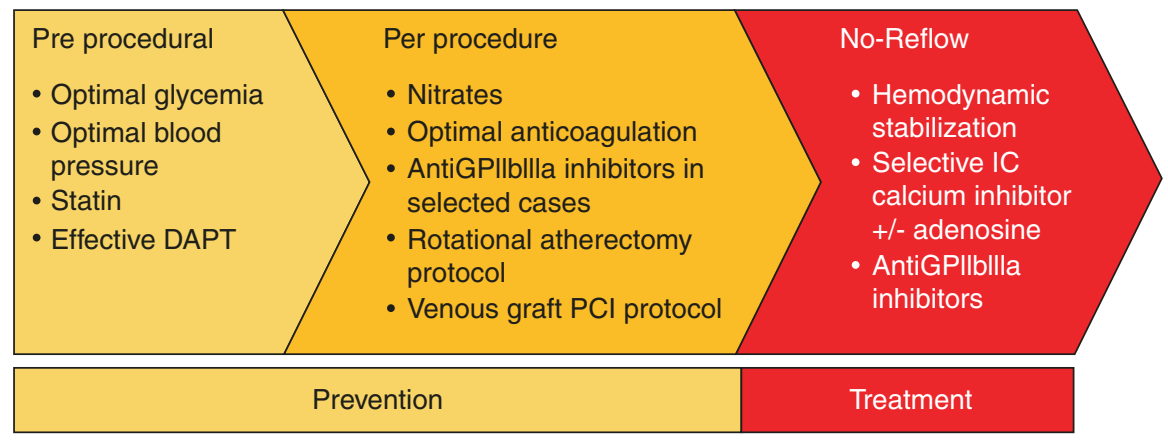

Fig. 17.1 No-reflow prevention and treatment 
complications is to perform short runs ( $15 \mathrm{~s}$ ) with a pecking motion to preserve flow at a speed rate of 140,000 rpm and avoid deceleration $>5000 \mathrm{rpm}$ with maintained patient haemodynamics [19].

\subsubsection{Venous Graft PCI}

Venous graft intervention is associated with higher rates of periprocedural $\mathrm{MI}$ and in-hospital mortality compared with PCI of native coronary arteries due to highly friable atherothrombotic debris of venous graft lesion. Therefore, distal embolization may result in slow or no-reflow phenomenon in more than $10 \%$ of cases. To prevent this distal embolization, it is recommended (IA) [20] to use distal protection device during stent implantation. In practice an American national registry showed that this protection device was used only in less than $25 \%$ of venous graft PCI, but still, the use of protection device was independently associated with a lower incidence of no-reflow but not in-hospital mortality [21]. It is interesting to notice this study evaluated the use of intragraft infusion of adenosine during peri procedure venous graft PCI. The study showed a significant reduction of no-reflow rate and increase average peak velocity compared to control group [22]. Nicardipine may be effective when administered before PCI in vein grafts to prevent no-reflow with minimal systemic depressant effect [18].

\subsubsection{No-Reflow Confirmation}

Priority is to exclude other mechanical aetiology which could occur after PCI and limit the contrast progression (Fig. 17.2). After PCI other causes such as coronary spasm, diffusion of coronary haematoma, coronary dissection, intracoronary occlusive thrombus or distal coronary stenosis could have similar angiographic and clinical presentation. Therefore, the easiest way to confirm this diagnosis is to perform,

Fig. 17.2 No-reflow diagnosis and management

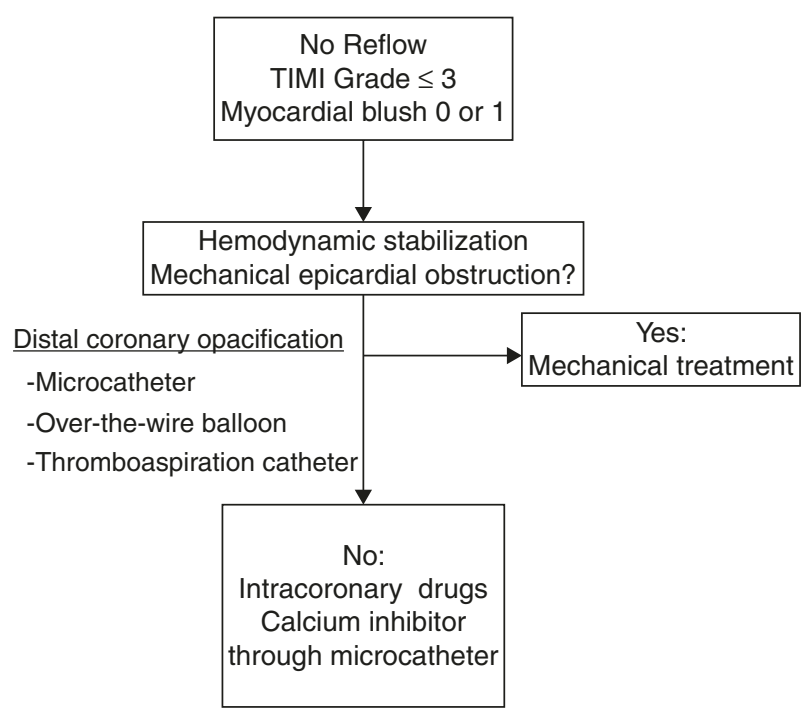



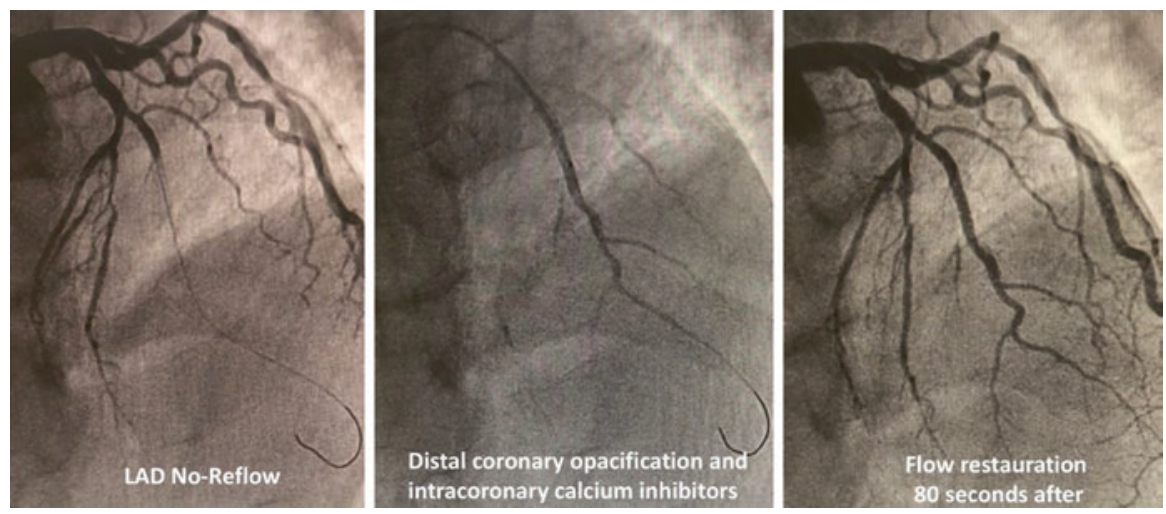

Fig. 17.3 Example of no-reflow management in a 72-year-old patient with anterior MI and noreflow after DES implantation in the mid-left anterior descending artery

after nitrates administration, distal coronary opacification using extension of guiding catheter, intracoronary microcatheter, thromboaspiration catheter or over-thewire angioplasty balloon. Careful and gentle distal contrast injection should be performed after aspirating some blood to confirm the true lumen position and the absence of obstruction due to thrombus or coronary wall at the exit of the catheter. Visualization of the distal part of the coronary artery is precious to exclude other causes of epicardial obstruction and confirm the no-reflow. Furthermore, microcatheters allow distal administration of drugs such as calcium inhibitors or adenosine which, by definition, could not reach the microvasculature in the no-reflow area from a guiding administration. An example is provided in Fig. 17.3.

\subsubsection{No-Reflow Treatment}

In no-reflow, microvascular damage is usually confined in the related coronary artery territory which constitutes a myocardial necrosis area. Therefore, treating no-reflow may not necessarily reduce the infarcted size but might improve blood flow to the necrotic area to improve area healing, infarction expansion and prevent left ventricular remodelling. Furthermore, restauration of flow will salvage the small vessels which may help promote collateral circulation and ensure drug delivery to the necrotic zone. To decrease the incidence of this phenomenon, short-term intracoronary and systemic drugs were studied to restore coronary flow within the no-reflow area (Table 17.1).

\subsubsection{Haemodynamic Stabilization}

Before starting dedicated therapeutics to treat no-reflow, it is essential to evaluate the patient haemodynamic to maintain optimal aortic blood pressure. Of note, noreflow of the right coronary artery is prone to reflex hypotension or bradycardia which needs atropine administration. General supportive measures are usually used to maintain stable haemodynamic such as fluid administration and if necessary 
Table 17.1 No-reflow aetiology, prevention and treatment

\begin{tabular}{|c|c|c|c|}
\hline & Aetiology & Prevention & Treatment \\
\hline \multirow[t]{2}{*}{$\begin{array}{l}\text { Myocardial } \\
\text { infarction }\end{array}$} & Thrombotic & $\begin{array}{l}\text { Optimal anticoagulation } \\
\text { Consider thrombectomy or } \\
\text { balloon inflation to restore TIMI } \\
\text { III flow with minimal invasive } \\
\text { strategy }\end{array}$ & \multirow[t]{2}{*}{$\begin{array}{l}\text { Anti-GPIIb/IIIa } \\
\text { Intracoronary } \\
\text { calcium inhibitors } \\
\text { Adenosine }\end{array}$} \\
\hline & $\begin{array}{l}\text { Microvascular } \\
\text { vasospasm }\end{array}$ & Nitrates & \\
\hline Rotablator & $\begin{array}{l}\text { Atherothrombotic } \\
\text { embolization } \\
\text { Platelet activation } \\
\text { Vasospasm }\end{array}$ & $\begin{array}{l}\text { Optimal anticoagulation } \\
\text { Nitrates } \\
\text { Maintained stable } \\
\text { haemodynamic (temporary } \\
\text { pacemaker/atropine in case of } \\
\text { severe bradycardia) } \\
\text { Flush infusion with nitrates and } \\
\text { or calcium inhibitors }\end{array}$ & $\begin{array}{l}\text { Intracoronary } \\
\text { calcium inhibitors } \\
\text { Adenosine }\end{array}$ \\
\hline Venous graft & Atherothrombotic & $\begin{array}{l}\text { Distal protection } \\
\text { Adenosine intra graft } \\
\text { Nicardipine }\end{array}$ & $\begin{array}{l}\text { Intragraft calcium } \\
\text { inhibitors } \\
\text { Adenosine }\end{array}$ \\
\hline Iatrogenic & Thrombotic & $\begin{array}{l}\text { Optimal anticoagulation with } \\
\text { ACT monitoring }\end{array}$ & $\begin{array}{l}\text { Heparin } \\
\text { anti-GPIIb/IIIa } \\
\text { Consider balloon } \\
\text { inflation or } \\
\text { thrombectomy }\end{array}$ \\
\hline
\end{tabular}

inotrope support such as epinephrine. In rare refractory cases intra-aortic balloon pump might be an option to maintain overall coronary perfusion.

\subsubsection{Thromboaspiration}

One must realize that manipulating the occluded thrombotic vessel with balloons and stents often results in distal embolization of the thrombus, which might contribute to no-reflow occurrence. To prevent distal embolization, thromboaspiration might help to reduce the thrombus burden and therefore the degree of no-reflow. Thromboaspiration was widely used in the past years in STEMI patients, but recent study results and metaanalyses failed to show benefit with an increased risk of stroke. Therefore, actual guidelines do not recommend to perform systematic thromboaspiration [23].

\subsubsection{Pharmacological Therapeutics}

\subsubsection{Adenosine}

Adenosine used in myocardial infarction might have some benefit in terms of preventing extensive microcirculation injury. Intravenous adenosine, given before reperfusion therapy, was suggested to reduce infarct size compared with placebo in the AMISTAD randomized clinical trial [24]. Similarly, the larger AMISTAD II trial demonstrated infarct size reduction in the adenosine group compared with the placebo group, but without significant benefit in terms of clinical outcome [25]. 
When looking at the post hoc analysis of the AMISTAD II trial, in the subgroup with successful reperfusion within $3 \mathrm{~h}$, the adjunct of adenosine infusion enhanced early and late survival and reduced the composite clinical endpoint of death or congestive heart failure at 6 months [26]. In addition, during reperfusion, the addition of intracoronary adenosine after thromboaspiration, through the thrombectomy catheter, showed a significant improvement in STR, with better 1-year left ventricular remodelling and reduction in clinical events compared with saline and nitroprusside $[27,28]$.

\subsubsection{Statins}

Based on STR, TIMI frame count and myocardial blush, Kim et al. showed that a high dose of atorvastatin may produce an optimal result in patients with STEMI undergoing PCI by improving microvascular myocardial perfusion, without significant clinical improvement [29].

\subsubsection{Calcium Inhibitors and Other Drugs}

Finally, intracoronary calcium inhibitors (verapamil, diltiazem and nicardipine) are probably the most evaluated and effective drugs available for the prevention and treatment of no-reflow phenomenon. In a meta-analysis by Su et al., including 7 trials involving 539 patients with intracoronary verapamil administration at a dosage of $200 \mu \mathrm{g}$ to $2 \mathrm{mg}$, the authors showed a significant decrease in no-reflow incidence, a better TIMI grade and frame count and a reduction in major adverse cardiac events (MACE), 2 months after PCI (relative risk 0.56, 95\% confidence interval $0.33-0.95$ ) [30]. Another Meta-analysis of 8 randomized controlled trials involving 494 patients evaluated the efficacy of the combination of verapamil and diltiazem or verapamil alone to treat no-reflow which suggested significant clinical benefit over standard of care with respect to no-reflow [31].

Nitroprusside is an effective intracoronary drug in the treatment of no-reflow. Two meta-analyses showed that intracoronary nitroprusside is beneficial in preventing no-reflow in reducing TIMI frame count and in improving left ventricular ejection fraction. It is also likely to reduce MACE $[32,33]$.

\subsection{Outcome}

After the procedure, non-invasive indexes are of importance to evaluate the myocardial damage and assess potential poor outcome.

\subsubsection{ECG}

Among several indices to assess microvascular obstruction with ECG, only the residual ST-segment elevation was an independent predictor of microvascular injury (odds ratio $19.1,95 \%$ confidence interval $2.4-154 ; P=0.005$ ) in multivariable analysis in a study evaluating ECG in 180 patients with STEMI. Interestingly, 
ST-segment resolution was not associated with LV function, infarct size, transmurality indexes or microvascular injury in multivariable analysis [34] (Fig. 17.4a). A distortion of the terminal portion of the QRS complex was significantly associated with infarct size, impaired myocardial salvage and reperfusion injury in 572 patients with reperfused STEMI as assessed by cardiac magnetic resonance imaging (CMR). Moreover, this QRS modification was independently associated with MACE [35].

\subsubsection{Echocardiography}

Myocardial contrast echocardiography (MCE) is a bedside technique that can be used to assess microvascular perfusion (Fig. 17.4c). Echo contrast agents are microbubbles of inert gases of sizes and rheology similar to that of red blood cells and can be administered intravenously. Myocardial uptake of microbubbles is delayed in areas of "no-reflow" and MVO. MCE is able to detect only 1/3 of patients with a no-reflow phenomenon after ACS [36, 37]. However, widespread use of MCE has been hampered by a long learning curve for image acquisition and reporting,
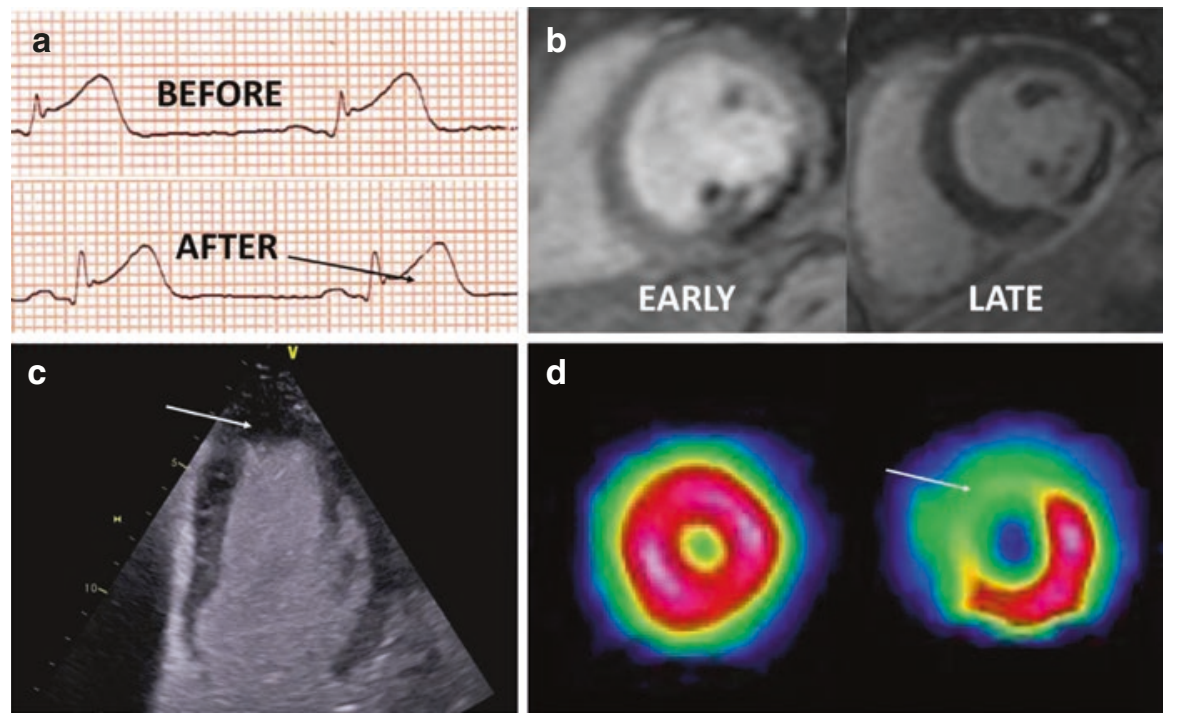

Fig. 17.4 Non-invasive tools to assess microvascular obstruction. (a) ST-segment resolution represents a useful tool of coronary microvascular obstruction after myocardial infarction. Black arrows showing absence of ST-segment resolution after artery recanalization. (b) Cardiac magnetic resonance. On late gadolinium enhancement, areas of microvascular obstruction are seen, hypoenhancement (so-called "dark zones") within an avidly enhancing site of myocardial infarction. (c) Myocardial contrast echocardiography showing lack of intra-myocardial contrast opacification (indicated by white arrow). (d) Single-photon emission computed tomography showing absent tracer uptake (white arrow, scintigraphic no-reflow phenomenon), as compared to normal uptake (left position). Adapted from Adjedj, J., et al. (2018). "Coronary microcirculation in acute myocardial ischaemia: From non-invasive to invasive absolute flow assessment." Arch Cardiovasc Dis 
uncertain reproducibility, concerns over microbubble contrast safety. Moreover, MCE has some limitations such as operator skills, moderate spatial resolution, incomplete left ventricular coverage and semi-quantitative assessment of MVO.

\subsubsection{Cardiac Magnetic Resonance}

CMR is the non-invasive gold standard to assess MVO. It allows multislice imaging with high tissue contrast and high spatial resolution, enabling accurate quantification and localization of $\mathrm{MVO}$ and the infarct size relative to the entire left ventricle (Fig. 17.4b). CMR-defined MVO has been well correlated with MCE, angiographic and invasive indices used for the assessment of MVO [38]. Symons et al. demonstrated that early post-infarction CMR-based MVO was a strong independent predictor of MACE in reperfused STEMI patients at long-term follow-up. Remarkably, MVO extent $\geq 2.6 \%$ of LV was the strongest independent predictor of death and heart failure hospitalization, overriding the prognostic performance of traditional outcome predictors and leading to better long-term risk stratification [39].

\subsubsection{Nuclear Imaging}

Both single-photon emission computed tomography [40] and positron emission tomography (PET) [41] demonstrated the "no-reflow" phenomenon in humans could be detected by nuclear imaging. Nuclear imaging no-reflow phenomenon can occur in a subgroup of patients without angiographic no-reflow phenomenon, that the myocardial damage depends on the severity of microvascular damage and that prolonged ischemia time may increase the likelihood of "microvascular no-reflow phenomenon" (Fig. 17.4d) [42]. However, PET scanning is still underutilized in clinical practice, and its clinical use is limited to sites with PET scans and cyclotrons or generators.

\section{Conclusion}

Currently, there is still a lack of an optimal treatment for no-reflow phenomenon. Prevention is effective to reduce no-reflow occurrence with medical therapy and proper use of dedicated techniques such as rotational atherectomy and venous graft PCI. The diagnosis of no-reflow remains a challenge and, if not recognized, may be treated inadvertently by additional PCI which will only harm the situation. When no-reflow occurs, the main objective is to diagnose properly, stabilize the patient and treat this condition by sub selective administration of vasodilator drugs to "open" the microcirculation, avoiding a systemic effect on the blood pressure. No-reflow management aims to improve coronary blood flow at the level of microcirculation to reduce myocardial damage and improve clinical outcome. 


\section{References}

1. Eeckhout E, Kern MJ. The coronary no-reflow phenomenon: a review of mechanisms and therapies. Eur Heart J. 2001;22:729-39.

2. Matsuo H, Watanabe S, Watanabe T, et al. Prevention of no-reflow/slow-flow phenomenon during rotational atherectomy - a prospective randomized study comparing intracoronary continuous infusion of verapamil and nicorandil. Am Heart J. 2007;154:994.e1-6.

3. Sharma SK, Dangas G, Mehran R, et al. Risk factors for the development of slow flow during rotational coronary atherectomy. Am J Cardiol. 1997;80:219-22.

4. Iwasaki K, Samukawa M, Furukawa H. Comparison of the effects of nicorandil versus verapamil on the incidence of slow flow/no reflow during rotational atherectomy. Am J Cardiol. 2006;98:1354-6.

5. Kaplan BM, Benzuly KH, Kinn JW, et al. Treatment of no-reflow in degenerated saphenous vein graft interventions: comparison of intracoronary verapamil and nitroglycerin. Catheter Cardiovasc Diagn. 1996;39:113-8.

6. Fischell TA, Carter AJ, Foster MT, et al. Reversal of "no reflow" during vein graft stenting using high velocity boluses of intracoronary adenosine. Catheter Cardiovasc Diagn. 1998;45:360-5.

7. Resnic FS, Wainstein M, Lee MK, et al. No-reflow is an independent predictor of death and myocardial infarction after percutaneous coronary intervention. Am Heart J. 2003;145:42-6.

8. Mehta RH, Harjai KJ, Boura J, et al. Prognostic significance of transient no-reflow during primary percutaneous coronary intervention for ST-elevation acute myocardial infarction. Am J Cardiol. 2003;92:1445-7.

9. Kunadian V, Harrigan C, Zorkun C, et al. Use of the TIMI frame count in the assessment of coronary artery blood flow and microvascular function over the past 15 years. J Thromb Thrombolysis. 2009;27:316-28.

10. Niccoli G, Cosentino N, Spaziani C, Fracassi F, Tarantini G, Crea F. No-reflow: incidence and detection in the cath-lab. Curr Pharm Des. 2013;19:4564-75.

11. Malmberg K, Ryden L, Efendic S, et al. Randomized trial of insulin-glucose infusion followed by subcutaneous insulin treatment in diabetic patients with acute myocardial infarction (DIGAMI study): effects on mortality at 1 year. J Am Coll Cardiol. 1995;26:57-65.

12. Iwakura K, Ito H, Ikushima M, et al. Association between hyperglycemia and the no-reflow phenomenon in patients with acute myocardial infarction. J Am Coll Cardiol. 2003;41:1-7.

13. Pantsios C, Kapelios C, Vakrou S, et al. Effect of elevated reperfusion pressure on "no reflow" area and infarct size in a porcine model of ischemia-reperfusion. J Cardiovasc Pharmacol Ther. 2016;21:405-11.

14. Li XD, Yang YJ, Hao YC, et al. Effect of pre-procedural statin therapy on myocardial no-reflow following percutaneous coronary intervention: a meta analysis. Chin Med J. 2013;126:1755-60.

15. Adgey AA. An overview of the results of clinical trials with glycoprotein IIb/IIIa inhibitors. Eur Heart J. 1998;19(Suppl D):D10-21.

16. Reisman M, Shuman BJ, Harms V. Analysis of heat generation during rotational atherectomy using different operational techniques. Cathet Cardiovasc Diagn. 1998;44:453-5.

17. Kini A, Reich D, Marmur JD, Mitre CA, Sharma SK. Reduction in periprocedural enzyme elevation by abciximab after rotational atherectomy of type B2 lesions: results of the Rota ReoPro randomized trial. Am Heart J. 2001;142:965-9.

18. Fischell TA, Maheshwari A. Current applications for nicardipine in invasive and interventional cardiology. J Invasive Cardiol. 2004;16:428-32.

19. Tomey MI, Kini AS, Sharma SK. Current status of rotational atherectomy. JACC Cardiovasc Interv. 2014;7:345-53.

20. Windecker S, Kolh P, Alfonso F, et al. 2014 ESC/EACTS Guidelines on myocardial revascularization: The Task Force on Myocardial Revascularization of the European Society of Cardiology (ESC) and the European Association for Cardio-Thoracic Surgery (EACTS) developed with the special contribution of the European Association of Percutaneous Cardiovascular Interventions (EAPCI). Eur Heart J. 2014;35:2541-619. 
21. Mehta SK, Frutkin AD, Milford-Beland S, et al. Utilization of distal embolic protection in saphenous vein graft interventions (an analysis of 19,546 patients in the American College of Cardiology-National Cardiovascular Data Registry). Am J Cardiol. 2007;100:1114-8.

22. Kapoor N, Yalamanchili V, Siddiqui T, Raza S, Leesar MA. Cardioprotective effect of highdose intragraft adenosine infusion on microvascular function and prevention of no-reflow during saphenous vein grafts intervention. Catheter Cardiovasc Interv. 2014;83:1045-54.

23. Ibanez B, James S, Agewall S, et al. 2017 ESC guidelines for the management of acute myocardial infarction in patients presenting with ST-segment elevation: the task force for the management of acute myocardial infarction in patients presenting with ST-segment elevation of the European Society of Cardiology (ESC). Eur Heart J. 2018;39(2):119-77.

24. Mahaffey KW, Puma JA, Barbagelata NA, et al. Adenosine as an adjunct to thrombolytic therapy for acute myocardial infarction: results of a multicenter, randomized, placebo-controlled trial: the acute myocardial infarction STudy of ADenosine (AMISTAD) trial. J Am Coll Cardiol. 1999;34:1711-20.

25. Ross AM, Gibbons RJ, Stone GW, Kloner RA, Alexander RW. A randomized, double-blinded, placebo-controlled multicenter trial of adenosine as an adjunct to reperfusion in the treatment of acute myocardial infarction (AMISTAD-II). J Am Coll Cardiol. 2005;45:1775-80.

26. Kloner RA, Forman MB, Gibbons RJ, Ross AM, Alexander RW, Stone GW. Impact of time to therapy and reperfusion modality on the efficacy of adenosine in acute myocardial infarction: the AMISTAD-2 trial. Eur Heart J. 2006;27:2400-5.

27. Niccoli G, Rigattieri S, De Vita MR, et al. Open-label, randomized, placebo-controlled evaluation of intracoronary adenosine or nitroprusside after thrombus aspiration during primary percutaneous coronary intervention for the prevention of microvascular obstruction in acute myocardial infarction: the REOPEN-AMI study (intracoronary nitroprusside versus adenosine in acute myocardial infarction). JACC Cardiovasc Interv. 2013;6:580-9.

28. Niccoli G, Spaziani C, Crea F. Left ventricular remodeling and 1-year clinical follow-up of the REOPEN-AMI trial. J Am Coll Cardiol. 2014;63:1454-5.

29. Kim JS, Kim J, Choi D, et al. Efficacy of high-dose atorvastatin loading before primary percutaneous coronary intervention in ST-segment elevation myocardial infarction: the STATIN STEMI trial. JACC Cardiovasc Interv. 2010;3:332-9.

30. Su Q, Li L, Liu Y. Short-term effect of verapamil on coronary no-reflow associated with percutaneous coronary intervention in patients with acute coronary syndrome: a systematic review and meta-analysis of randomized controlled trials. Clin Cardiol. 2013;36:E11-6.

31. Wang L, Cheng Z, Gu Y, Peng D. Short-term effects of verapamil and diltiazem in the treatment of no reflow phenomenon: a meta-analysis of randomized controlled trials. Biomed Res Int. 2015;2015:382086.

32. Su Q, Li L, Naing KA, Sun Y. Safety and effectiveness of nitroprusside in preventing no-reflow during percutaneous coronary intervention: a systematic review. Cell Biochem Biophys. 2014;68:201-6.

33. Zhao S, Qi G, Tian W, Chen L, Sun Y. Effect of intracoronary nitroprusside in preventing no reflow phenomenon during primary percutaneous coronary intervention: a meta-analysis. J Interv Cardiol. 2014;27:356-64.

34. Nijveldt R, van der Vleuten PA, Hirsch A, et al. Early electrocardiographic findings and MR imaging-verified microvascular injury and myocardial infarct size. JACC Cardiovasc Imaging. 2009;2:1187-94.

35. Rommel KP, Badarnih H, Desch S, et al. QRS complex distortion (grade 3 ischaemia) as a predictor of myocardial damage assessed by cardiac magnetic resonance imaging and clinical prognosis in patients with ST-elevation myocardial infarction. Eur Heart J Cardiovasc Imaging. 2016;17:194-202.

36. Dwivedi G, Janardhanan R, Hayat SA, Lim TK, Greaves K, Senior R. Relationship between myocardial perfusion with myocardial contrast echocardiography and function early after acute myocardial infarction for the prediction of late recovery of function. Int $\mathrm{J}$ Cardiol. 2010;140:169-74. 
37. Galiuto L, Garramone B, Scara A, et al. The extent of microvascular damage during myocardial contrast echocardiography is superior to other known indexes of post-infarct reperfusion in predicting left ventricular remodeling: results of the multicenter AMICI study. J Am Coll Cardiol. 2008;51:552-9.

38. Nijveldt R, Beek AM, Hirsch A, et al. Functional recovery after acute myocardial infarction: comparison between angiography, electrocardiography, and cardiovascular magnetic resonance measures of microvascular injury. J Am Coll Cardiol. 2008;52:181-9.

39. Symons R, Pontone G, Schwitter J, et al. Long-term incremental prognostic value of cardiovascular magnetic resonance after ST-segment elevation myocardial infarction: a study of the collaborative registry on CMR in STEMI. JACC Cardiovasc Imaging. 2018;11(6):813-25. https://doi.org/10.1016/j.jcmg.2017.05.023.

40. Schofer J, Montz R, Mathey DG. Scintigraphic evidence of the "no reflow" phenomenon in human beings after coronary thrombolysis. J Am Coll Cardiol. 1985;5:593-8.

41. Jeremy RW, Links JM, Becker LC. Progressive failure of coronary flow during reperfusion of myocardial infarction: documentation of the no reflow phenomenon with positron emission tomography. J Am Coll Cardiol. 1990;16:695-704.

42. Kondo M, Nakano A, Saito D, Shimono Y. Assessment of "microvascular no-reflow phenomenon" using technetium-99m macroaggregated albumin scintigraphy in patients with acute myocardial infarction. J Am Coll Cardiol. 1998;32:898-903.

Open Access This chapter is licensed under the terms of the Creative Commons Attribution 4.0 International License (http://creativecommons.org/licenses/by/4.0/), which permits use, sharing, adaptation, distribution and reproduction in any medium or format, as long as you give appropriate credit to the original author(s) and the source, provide a link to the Creative Commons license and indicate if changes were made.

The images or other third party material in this chapter are included in the chapter's Creative Commons license, unless indicated otherwise in a credit line to the material. If material is not included in the chapter's Creative Commons license and your intended use is not permitted by statutory regulation or exceeds the permitted use, you will need to obtain permission directly from the copyright holder.

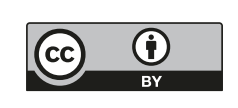

